\title{
EPO attenuates inflammatory cytokines by Muller cells in diabetic retinopathy
}

\author{
Xia Lei ${ }^{1,2,3}$, Jingfa Zhang ${ }^{2}$, Jianfeng Shen ${ }^{1,3}$, Liu-Mei Hu ${ }^{4}$, Yalan $\mathrm{Wu}^{1}$, Lisha Mou ${ }^{1,3}$, Guoxu Xu ${ }^{5}$, Weiye Li ${ }^{2,4,6}$, Guo-Tong \\ $\mathrm{Xu}^{1,2}$
}

${ }^{I}$ Institute of Health Sciences (IHS), Shanghai Institutes for Biological Sciences (SIBS), Chinese Academy of Sciences (CAS) and Shanghai Jiao Tong University School of Medicine (SJTUSM), Shanghai, China, ${ }^{2}$ Tongji Eye Institute and Department of Regenerative Medicine, Tongji University School of Medicine, Shanghai, China, ${ }^{3}$ Graduate School of Chinese Academy of Sciences, Beijing, China, ${ }^{4}$ Department of Ophthalmology, Peking Union Medical College Hospital, Chinese Academy of Medical Sciences, Beijing, China, ${ }^{5}$ Department of Ophthalmology, Second Affiliated Hospital of Soochow University, Suzhou, China, ${ }^{6}$ Department of Ophthalmology, Drexel University College of Medicine, Philadelphia, PA, USA

\section{TABLE OF CONTENTS}

\author{
1. Abstract \\ 2. Introduction \\ 3. Materials and methods \\ 3.1. Muller cell culture \\ 3.2. Experimental animals and intravitreal EPO treatment \\ 3.3. Morphological examination and MTT assay \\ 3.4. In situ detection of cell death by TUNEL assay \\ 3.5. RNA purification and real-time $P C R$ \\ 3.6. ELISA \\ 3.7. Luciferase assay for $A P-1$ and NF-kappaB activity \\ 3.8. Immunostaining \\ 4. Results \\ 3.9. Statistical analysis \\ 4.1. Effects of glyoxal on $\mathrm{rMC}-1$ cells \\ 4.2. Suppression by EPO on $m R N A$ levels of TNF-alpha and IL-1beta but not IL-6 and VEGF \\ 4.3. Attenuation by EPO on secretion of TNF-alpha and IL-1beta but not IL-6 and VEGF \\ 4.4. Involvement of EPO/EPOR system in the inflammatory response \\ 4.5. Modulation of AP-1 but not NF-kappaB activity by glyoxal and EPO \\ 4.6. Attenuation of TNF-alpha and IL-1beta production by EPO in diabetic rats \\ 5. Discussion \\ 6. Acknowledgement \\ 7. References
}

\section{ABSTRACT}

Diabetic retinopathy (DR) is a chronic, low-grade inflammatory disease. We aimed to investigate the regulatory effects of erythropoietin (EPO) on the inflammatory cytokine production by Muller cells under the condition of DR. The expression levels of TNF-alpha, IL-1beta, IL-6 and VEGF in cultured rat Muller cells were enhanced by $1 \mathrm{mM}$ glyoxal. The elevated TNF-alpha and IL-1beta, but not IL-6 and VEGF, were decreased by 2 $\mathrm{U} / \mathrm{ml}$ EPO as detected by real-time PCR and ELISA. Moreover, the activity of AP-1 but not NF-kappaB was modulated by glyoxal and EPO. Intravitreal injection of EPO performed $24 \mathrm{~h}$ prior to sacrifice significantly reduced TNF-alpha and IL-1beta production while moderately attenuating IL-6 and VEGF in the retinas of streptozotocininduced diabetic rats. Furthermore, Muller cells were identified as the main source of IL-1beta production as indicated by co-localization of IL-1beta and CRALBP in situ. These findings implicate therapeutic potential of EPO in the amelioration of inflammation in diabetic retinas.

\section{INTRODUCTION}

Diabetic retinopathy (DR) is the most common microvascular complication in patients with diabetes and may lead to visual impairment and even blindness (1). Although the exact mechanisms by which elevated glucose or other factors initiate the vascular disruption in diabetic retinas remain poorly defined, several biochemical pathways have been implicated in the pathogenesis. One well-characterized pathway is the formation of advanced glycation end products (AGEs) that are capable of irreversibly modifying the chemical properties and functions of diverse key molecules (2). Glyoxal (GO), an intermediate in AGEs formation, has been demonstrated in several studies on the diabetes-associated retinal abnormalities to be a powerful inducer of cell apoptosis (3, 4). More recently, it is reported that in human vascular endothelial cells glyoxal causes inflammatory injury (5). Accumulating evidence suggests that the up-regulation of inflammatory factors such as TNF-alpha, IL-1beta, IL-6 and VEGF contributes to the blood-retinal barrier (BRB) 
breakdown in the development of DR (6-9). Moreover, Muller cells, the principal glial cells of the retina, associated with the inflammatory process in vivo have been demonstrated by using an immortalized rat Muller cell line (rMC-1) in vitro $(10,11)$. Therefore, the present study was designed to examine whether glyoxal could induce inflammatory response and the profile of cytokine expression in rMC-1 cells.

The anti-inflammatory effect of erythropoietin (EPO) has been well documented in a variety of animal models of neurological diseases such as traumatic and ischemic injuries in the brain and spinal cord, and multiple sclerosis (MS) (12-17). For instance, in a rat model of middle cerebral artery ischemia reperfusion, EPO significantly reduces the activation of astrocytes as well as the recruitment of leukocytes and microglia into the infarction area, and down-regulates the production of TNFalpha and IL-6 (13). Based on a study on experimental autoimmune encephalitis (EAE), an animal model of MS, EPO markedly reduces the levels of IL- 6 in acute rat models (16). EPO also decreases the secretion of TNFalpha and IL-1beta in the spinal cord in chronic murine autoimmune encephalomyelitis models (16, 18). Furthermore, our previous studies revealed that intravitreal injection of EPO could preserve the BRB function in streptozotocin (STZ)-induced diabetic rats (19). In addition, encouraging results on the efficacy of intravitreal EPO treatment have been obtained from our recent clinical series in patients with chronic and progressive diabetic macular edema (20), prompting us to elucidate the underlying molecular mechanisms, including the possible modulation of inflammatory factors by EPO.

Nuclear factor-kappaB (NF-kappaB) and activator protein 1 (AP-1) are key transcription factors that may modulate production of inflammatory mediators (21). It has been demonstrated that the cardioprotection of EPO pretreatment is due in part to the suppression of the inflammatory response via down-regulation of NF-kappaB and AP-1 induced by ischemia-reperfusion injury (22). Therefore, we have studied the induction of these two factors in our in vitro model.

To determine whether an inflammatory response could be induced by AGEs and regulated by EPO in retinal Muller cells under diabetes-like conditions, we used both rMC-1 cells stimulated with glyoxal at concentrations below the cytotoxic level and diabetic rats induced by STZ as the experimental models for the current study. We found that EPO attenuated glyoxal-induced inflammation of Muller cells both in vitro and in vivo, which may be due to the suppression of AP-1 activation and in turn the downregulation of the downstream inflammatory cytokines such as TNF-alpha and IL-1beta.

\section{MATERIALS AND METHODS}

\subsection{Muller cell culture}

The rMC-1 cells were kindly supplied by Vijay Sarthy (Northwestern University, Evanston, IL). The cells were maintained in normal glucose $(5 \mathrm{mM})$ Dulbecco's modified Eagle's Medium (DMEM) containing 10\% fetal bovine serum (FBS) (High Clone Corporation, Logan, Utah) and $1 \%$ penicillin/streptomycin (Invitrogen, Carlsbad, CA) at $37^{\circ} \mathrm{C}$ with $5 \% \mathrm{CO}_{2}$ in a humidified incubator. When the cells reached $70 \%$ confluence, they were starved in medium without FBS for $16 \mathrm{~h}$, and then exposed to glyoxal (Sigma-Aldrich, St. Louis, MO) alone or combined with EPO ( $\mathrm{R}$ and D Systems, Minneapolis, $\mathrm{MN}$ ) for $12 \mathrm{~h}$. To determine the effect of EPO on the induction of AP-1 transcription factor, cells were pretreated with $250 \mathrm{ng} / \mathrm{ml}$ recombinant human soluble EPO receptor (sEPOR, R and D Systems, Minneapolis, MN) or $1 \mu \mathrm{M}$ nordihydroguaiaretic acid (NDGA, Cayman Chemicals, Ann Arbor, MI) for $1 \mathrm{~h}$ before the incubation of glyoxal and EPO.

\subsection{Experimental animals and intravitreal EPO treatment}

Male Sprague-Dawley rats weighing 120-160 g (Slaccas, SIBS, Shanghai, China) were used. They were treated according to The Guide for the Care and Use of Animals (National Research Council and Tongji University). Diabetes induction and intravitreal injections were described previously (19). Briefly, for diabetes induction, a single intraperitoneal injection of STZ (in citric buffer, $\mathrm{pH} \mathrm{4.5)}$ at a dose of $60 \mathrm{mg} / \mathrm{kg}$ body weight was performed after they had been fasted for $24 \mathrm{~h}$. The control rats received an equal volume of citric buffer. All animals were maintained in a $12 \mathrm{~h}$ alternating light-dark cycle, and allowed to eat and drink ad libitum. Rats receiving STZ were declared diabetic when their blood glucose exceeded $250 \mathrm{mg} / \mathrm{dl}$ for three consecutive days. The rats were excluded from the experiment if they failed to develop diabetes. Two or twenty weeks after the onset of diabetes, they were killed. One day prior to sacrifice, the diabetic rats were randomly divided into two groups: EPO treated and saline treated. EPO $(\mathrm{r}-\mathrm{Hu}-\mathrm{EPO}, 0.1 \mu \mathrm{g} / \mu \mathrm{l}$ in normal saline, i.e., $153 \mathrm{U} / \mathrm{mg}$; Sigma-Aldrich, St. Louis, MO) was diluted to $4 \mathrm{mU} / \mu \mathrm{l}$. Intravitreal injection of $2 \mu \mathrm{l}(8 \mathrm{mU}) \mathrm{EPO}$ per eye was performed, with a 30 -gauge, 0.5 -in. needle (BD Biosciences, Franklin Lakes, NJ) on a microsyringe (Hamilton, Reno, NV), using a temporal approach, $2 \mathrm{~mm}$ posterior and parallel to the limbus. Sham injections $(2 \mu \mathrm{l}$ normal saline) were performed to both non-diabetic control rats as well as the saline diabetic rats. The rats recovered spontaneously from the anesthesia and then were sent back to the animal room with food and water ad libitum.

\subsection{Morphological examination and MTT assay}

The morphological changes of rMC-1 cells after glyoxal treatment were examined under light microscope (Nikon, Eclipsnet VSL, Japan) equipped with digital camera.

For survival assays, rMC-1 cells were plated at a density of $1.5 \times 10^{4}$ per well on 96 well-plates to determine the cytotoxic effects of glyoxal. After incubation for $24 \mathrm{~h}$ in full medium, the cells were deprived of neurotrophins by medium change without FBS for $16 \mathrm{~h}$, and then cultures were supplemented with glyoxal at varying concentrations for $12 \mathrm{~h}$. Control and glyoxal treated cells were incubated in serum-free medium containing $0.5 \mathrm{mg} / \mathrm{ml}$ MTT (Sigma- 
Aldrich, St. Louis, MO) for $4 \mathrm{~h}$. The media were then removed and the formazan crystals produced in the wells were dissolved in $150 \mu \mathrm{l}$ dimethylsulfoxide (DMSO). The absorbance was measured at $570 \mathrm{~nm}$ with background subtraction at $630 \mathrm{~nm}$ using a microplate spectrophotometer (Tecan, Crailsheim, Germany). The cell viability was expressed as a percentage of untreated controls, which were defined as $100 \%$ for each experiment.

\subsection{In situ detection of cell death by TUNEL assay}

Evaluation of apoptotic cells was performed using a TUNEL assay (In situ Cell Death Detection Kit; Roche Diagnostics, Mannheim, Germany) according to the manufacture's instruction. Briefly, rMC-1 cells were cultured on coverslips. After treatment with glyoxal $(0,1,2$ $\mathrm{mM}$ ) for $12 \mathrm{~h}$, cells were washed twice with PBS, and then fixed and permeabilized in $0.1 \%$ Triton X-100 in PBS for 5 min. TUNEL reaction mixture was prepared by adding terminal deoxynucleotidyl transferase to nucleotide mixture. Then, each coverslip was incubated with $50 \mu \mathrm{l}$ of this mixture in a humidified chamber at $37^{\circ} \mathrm{C}$ in dark for 60 min. After rinsing with PBS, nuclei were counterstained with DAPI (4, 6-diamidino-2-phenylindole). Finally, they were analyzed under a fluorescence microscope. Positive controls were coverslips that had been treated with recombinant DNase I for $10 \mathrm{~min}$ at room temperature before the labeling procedure. Negative controls were the samples treated with $10 \mu \mathrm{l}$ label solution without terminal transferase. The apoptotic index (\%) was calculated as the number of TUNEL-positive stained cells divided by the total cell number counted by Image-Pro software.

\subsection{RNA purification and real-time $P C R$}

Total RNA was isolated from rMC-1 cells using TRIZOL Reagent (Trizol; Life Technologies, Grand Island, NY) and the reverse transcribed product was amplified by PCR using SYBR Green Real-time PCR master mix (ToYoBo, Osaka, Japan). The reactions were carried out on an ABI/PRISM 7900 HT sequence detector system (Applied Biosystems, Foster City, CA) for 40 cycles $\left(95^{\circ} \mathrm{C}\right.$ for $15 \mathrm{sec}, 60^{\circ} \mathrm{C}$ for $15 \mathrm{sec}, 72^{\circ} \mathrm{C}$ for $45 \mathrm{sec}$ ) after an initial $30 \mathrm{sec}$ of incubation at $95^{\circ} \mathrm{C}$. After amplification, melting curve analyses were performed to control the specificity of the reactions. Reactions were performed in triplicate and the values were normalized against beta-actin. Relative levels of gene expression changes were calculated by 2DDCt method. Results were presented as mean fold change in mRNA expression of treated cells compared with untreated cells in four to six independent experiments. The sequences of PCR primers are given below. TNF-alpha (10): 5'-CCTTATCTACTCCCAGGTTCT C-3' and 5'TTTCTCCTGGTATGAAATGGC-3'. IL-1beta (10): 5'CAGAAGAATCTAGTTGTCC-3'

TCATAAACACTCTCATCCACAC-3'. IL-6: 5'TATGAACAGCGATGATGCACTG-3' and 5'TTGCTCTGAATGACTCTGGCTT-3'. VEGF: 5'CCTGGTGGACATCTTCCAGGAGTACC-3' and 5'GAAGCTCATCTCTCCTATGTGCTGGC-3'. EPO: 5'CTGCTTCGGGT GCTGGGAGC-3' and 5'CGGGTACACGGCAGTGGCAG-3'. EPOR: 5'CATCCCGGGGCTCCGAAGAACTT-3' and 5'GTCCGCGGTCGGCAGTGAACAC-3'. Beta-actin: 5'-
GTAAAGACCTCTATGCCAACA-3,

and

5'GGACTCATCGTACTCCTGCT-3'.

\subsection{ELISA}

After treatment as indicated in the text, the cell culture supernatants were removed and retained at $-70^{\circ} \mathrm{C}$. For rats, individual retinas from experimental and control groups ( 6 single retinas from 6 rats selected randomly per group) were isolated and homogenized in ice-cold radioimmune precipitation assay (RIPA) buffer $(10 \mathrm{mM}$ Tris- $\mathrm{HCl}(\mathrm{pH} 7.5), 150 \mathrm{mM} \mathrm{NaCl}, 0.1 \%$ sodium dodecyl sulfate (SDS), $1 \%$ Nonidet $\mathrm{P}-40$, and $1 \%$ sodium deoxycholate) for ELISA. This buffer was supplemented with the protease inhibitor PMSF. After 15 min incubation on ice, the extracts were clarified by centrifugation at $12,000 \mathrm{~g}$ for $15 \mathrm{~min}$ at $4^{\circ} \mathrm{C}$ and stored at $-70^{\circ} \mathrm{C}$. The concentrations of TNF-alpha, IL-1beta, IL-6 and VEGF were measured with ELISA kits ( $\mathrm{R}$ and $\mathrm{D}$ Systems, Minneapolis, MN). The minimum detectable dose of rat TNF-alpha and IL-1beta is typically less than $5 \mathrm{pg} / \mathrm{ml}$. The minimum detectable dose of rat IL-6 and VEGF were 21 $\mathrm{pg} / \mathrm{ml}$ and $8.4 \mathrm{pg} / \mathrm{ml}$, respectively. The protein concentration of cells was measured with BCA Protein Assay Kit (Pierce, Rockford, IL). Values were compared with the standard curve of individual cytokines, normalized to total protein concentrations, and expressed as picogram per milligram of protein.

\subsection{Luciferase assay for AP-1 and NF-kappaB activity}

The rMC-1 cells were plated in a 96-well plate, co-transfected using lipofectamine 2000 (Invitrogen, Carlsbad, CA) with $500 \mathrm{ng}$ pNF-kappaB-luc or $500 \mathrm{ng}$ pAP-1-luc (firefly luciferase) and $10 \mathrm{ng}$ internal control plasmid pRL-CMV (renilla luciferase) for normalization of transfection efficiency. After $24 \mathrm{~h}$, the cells were incubated in serum-free medium and treated with $1 \mathrm{mM}$ glyoxal alone or combined with $2 \mathrm{U} / \mathrm{ml}$ EPO for $12 \mathrm{~h}$. Renilla luciferase and firefly luciferase were measured (Dual Luciferase Assay System; Promega Corporation, Madison, WI) and results were presented as the ratio of firefly luciferase activity.

\subsection{Immunostaining}

The rMC-1 cells were grown on coverslips with desired treatments. They were fixed with $4 \%$ paraformaldehyde for $15 \mathrm{~min}$, permeabilized with $0.2 \%$ Triton X-100 in PBS for 5 min, and blocked with 1\% BSA in PBS for $1 \mathrm{~h}$. After that, they were stained with anti-EPO antibody (1:50; H-162, Santa Cruz Biotechnology), antip65 antibody (1:50; C-20, Santa Cruz Biotechnology, Santa Cruz, CA) and cy3-conjugated anti-rabbit IgG, and then visualized and photographed under the fluorescence microscope.

Cryostat sections of rat retinas were prepared as described previously (19). Briefly, the eyes were fixed in PBS-buffered $4 \%$ paraformaldehyde for $24 \mathrm{~h}$ and then opened along the ora serrata, and the posterior eyecups were dehydrated through a gradient concentration of sucrose from $10 \%$ to $30 \%$. After dehydration, the eyecups were embedded in OCT compound (Tissue Tek, Sakura) for sectioning. $10 \mu \mathrm{m}$-thick sections were co-incubated 

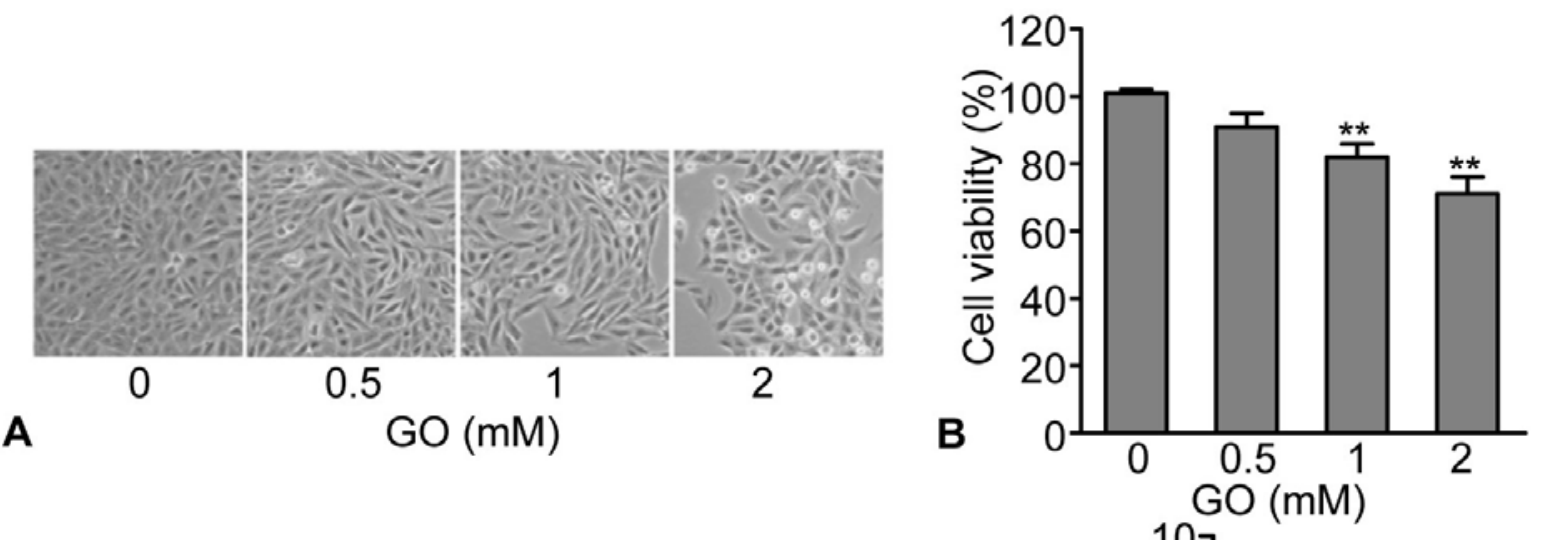

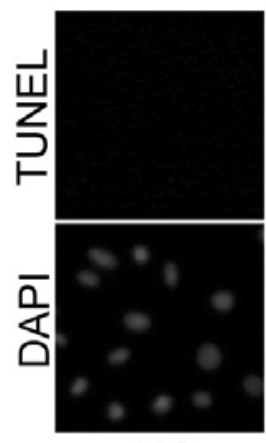

C

NC

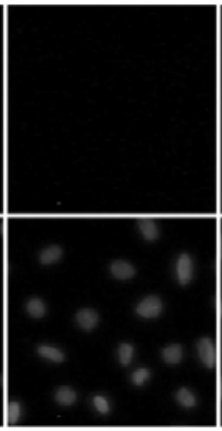

0

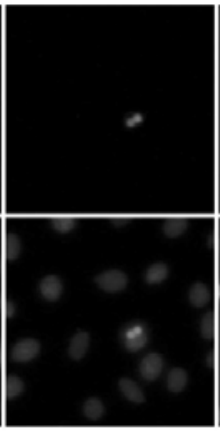

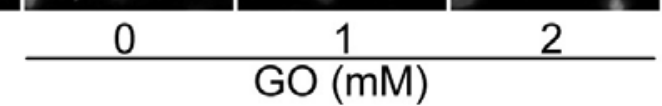

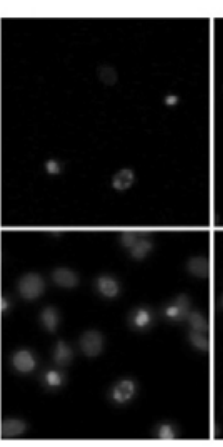

2

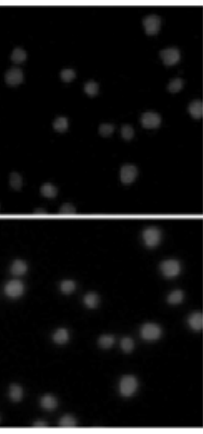

PC

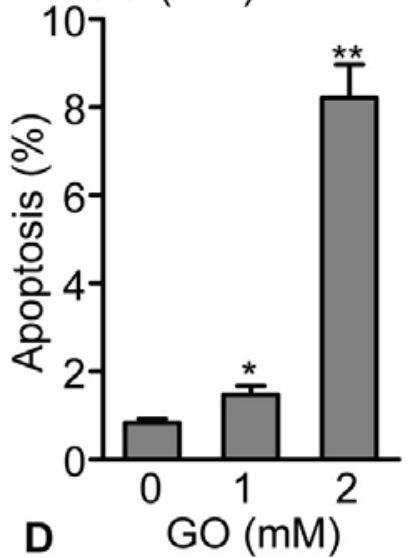

Figure 1. Effects of glyoxal on rMC-1 cells. The alterations in cell morphology, viability and apoptosis were evaluated after the treatment with varying concentrations of glyoxal for $12 \mathrm{~h}$. A: Concentration-dependent cytotoxic effects of glyoxal $(0,0.5,1,2$ $\mathrm{mM})$ were observed in the bright field images. Magnification: $\times 100$. B: Effects of glyoxal on cell viability were assessed by MTT assay. Results are expressed as percentage of control (without glyoxal). Data (mean $+/-$ SE) are based on five independent experiments for each group. $(* * P<0.01 v s$ cont $) \mathrm{C}$ : Apoptotic cells were detected after exposure to glyoxal $(0,1,2 \mathrm{mM})$ by fluorescence microscopy after TUNEL staining. Total number of cells in a given area was determined with DAPI nuclear staining. NC: negative control; PC: positive control. Magnification: $\times 100$. D: Apoptosis ratio was obtained by percentage of labeled cells to total cells in the same area from 9 random fields. Apoptotic cells increased significantly at $2 \mathrm{mM}$. $(* P<0.05$, $* * P<0.01, \mathrm{n}=9)$

with anti-IL-1beta antibody (1:50; H-153, Santa Cruz Biotechnology, Santa Cruz, CA) and anti-CRALBP antibody (1:100; ab15051, Abcam, Cambridge, MA), then stained with the mixture of two secondary antibodies (cy3conjugated anti-rabbit IgG and FITC-conjugated antimouse IgG). After nuclear staining using DAPI, they were washed with PBS, mounted using Dako Cytomation Fluorescent mounting medium (Dako A/S, Glostrup, Denmark) and observed under the fluorescence microscope.

\subsection{Statistical analysis}

Quantitative data are presented as mean $+/-$ SE. The data from real-time PCR were based on 4-6 independent experiments in rMC-1 cells or individual rat retinas. Reactions were carried out in triplicate. The samples for ELISA were collected from rMC-1 cell supernatants in 4 independent experiments or individual retinas of 6 rats. The statistical evaluations were performed using ANOVA followed by Bonferroni post-hoc test for multiple comparisons or Student's $t$-test. A $P$-value of 0.05 or less was considered statistically significant.

\section{RESULTS}

\subsection{Effects of glyoxal on $\mathrm{rMC}-1$ cells}

To evaluate the direct effects of glyoxal on retinal Muller cells, we first conducted morphological examinations in cultured rMC-1 cells after $12 \mathrm{~h}$ treatment with various concentrations of glyoxal. In the bright-field images, there were no obvious signs of toxicity at low concentrations $(0.5 \mathrm{mM})$. However, a slight decrease in cell density (at $1 \mathrm{mM}$ glyoxal) and a remarkable increase in cell death $(2 \mathrm{mM})$ were observed at high concentrations of glyoxal (Figure 1A). Consistent with the morphological changes, glyoxal treatment caused the reduction of cell viability in a dose-dependent manner, as measured by MTT assay (Figure 1B). In the presence of $1 \mathrm{mM}$ or $2 \mathrm{mM}$ glyoxal, the viability of rMC-1 cells was suppressed to $82 \%$ or $71 \%$ as compared with untreated cells, respectively. 

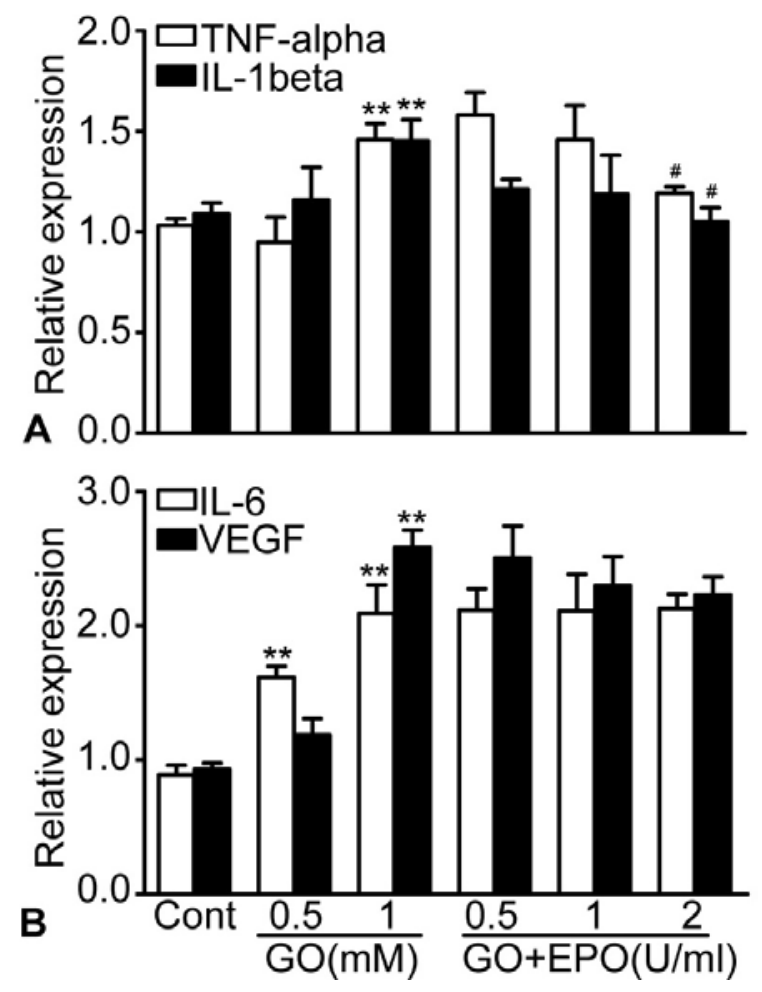

Figure 2. Relative expression levels of TNF-alpha, IL1beta (A), IL-6 and VEGF (B) in rMC-1 cells were determined after stimulation with various concentrations of glyoxal $(0.5,1 \mathrm{mM})$ or $1 \mathrm{mM}$ glyoxal in combination with EPO $(0.5,1,2 \mathrm{U} / \mathrm{ml})$ for $12 \mathrm{~h}$ by real-time PCR and normalized with respect to beta-actin. The expression of TNF-alpha and IL-1beta were almost normalized by the addition of $2 \mathrm{U} / \mathrm{ml}$ EPO. $(* * P<0.01$ vs Cont; $\# P<0.05$ vs 1 $\mathrm{mM} \mathrm{GO} ; \mathrm{n}=4-6$ )

We next tried to identify whether the cell death observed in rMC-1 cells following exposure to glyoxal was apoptotic (Figure 1C). After TUNEL staining, substantially more positive cells characterized by nuclear condensation and fragmentation were seen at the concentration of $2 \mathrm{mM}$ than at $1 \mathrm{mM}$. Likewise, the percentage of apoptotic cells dramatically increased to $8.2 \%$ at $2 \mathrm{mM}$, while it was only $1.5 \%$ at $1 \mathrm{mM}$ (Figure 1D). Therefore, we chose the relatively low concentrations ( $1 \mathrm{mM}$ or less) for the further experiments on inflammatory response because glyoxal at these concentrations had minor effect on cell apoptosis.

\subsection{Suppression by EPO on mRNA levels of TNF-alpha and IL-1beta but not IL-6 and VEGF}

We investigated whether the decrease in cell viability after glyoxal treatment was associated with inflammatory response. Relative mRNA expression of four inflammatory genes was assessed by real-time PCR (Figure 2). There was no significant change induced by glyoxal at the concentration of $0.5 \mathrm{mM}$ in the expression of TNFalpha, IL-1beta and VEGF. However, at $1 \mathrm{mM}$, a modest increase in TNF-alpha and IL-1beta (1.5 folds) and a remarkable elevation in VEGF (2.6 folds) were detected. Besides, IL-6 expression was increased in a dose-dependent fashion after stimulation with glyoxal $(0.5 \mathrm{mM}, 1.6$ folds; 1 $\mathrm{mM}, 2.1$ folds). Based on these results, we determined to use $1 \mathrm{mM}$ glyoxal for $12 \mathrm{~h}$ in rMC-1 cells in the following studies on the expression of inflammatory genes.

EPO has been reported as an anti-inflammatory factor in a range of different cell culture models (23-26). Here, we inspected whether EPO could reduce the inflammatory gene expression induced by glyoxal in rMC1 cells. Three different doses of EPO $(0.5,1,2 \mathrm{U} / \mathrm{ml})$ were added into the medium containing glyoxal, and the efficacy was investigated (Figure 2). Results of real-time PCR showed that TNF-alpha expression was down-regulated gradually by the increasing doses of EPO (1.2 folds at 2 $\mathrm{U} / \mathrm{ml}$ ). For IL-1beta, EPO exerted a more powerful inhibitory effect particularly at dose of $2 \mathrm{U} / \mathrm{ml}$, in which the expression level was almost the same as that in untreated cells. In contrast, with the addition of EPO at all of these doses, no influence on IL-6 expression was displayed. No significant change in VEGF was detected under the treatment of EPO at $2 \mathrm{U} / \mathrm{ml}$. These results indicated that EPO at appropriate doses could selectively suppress the mRNA expression of these inflammatory genes.

\subsection{Attenuation by EPO on secretion of TNF-alpha and IL-1beta but not IL-6 and VEGF}

Because all the four inflammatory factors can be actively secreted by inflammatory cells, we then tried to find out whether EPO could block the secretion of them in rMC-1 cells. After the incubation in the presence of $1 \mathrm{mM}$ glyoxal, with or without the addition of $2 \mathrm{U} / \mathrm{ml}$ EPO for 12 $\mathrm{h}$, the supernatants of the cells were collected and the amount of TNF-alpha, IL-1beta, IL-6 and VEGF was measured by ELISA (Figure 3). With glyoxal treatment, dramatic increase was detected in the secretion of IL-1beta (139.4 vs 27.0) and VEGF (1732.5 vs 190.3), to a lesser extent in TNF-alpha (34.0 vs 15.0), and the least in IL-6 (648.3 vs $508.7 \mathrm{pg} / \mathrm{mg}$ protein). In addition, the inhibitory effects of EPO on cytokine secretion were also verified in TNF-alpha (19.6) and IL-1beta (67.2), while no obvious variation was found in IL-6 (625.9) and VEGF (1762.8 $\mathrm{pg} / \mathrm{mg}$ protein). Thus, these results suggested that the production of TNF-alpha and IL-1beta but not IL-6 and VEGF could be attenuated by EPO administration in the glyoxal model of rMC-1 cells.

\subsection{Involvement of EPO/EPOR system in the inflammatory response}

Since Muller cells have been identified as the main source of EPO production in rat retina (27), we investigated whether endogenous EPO was involved in this glyoxalinduced inflammatory response. The expression of EPO was up-regulated about 1.3 folds after exposure to $1 \mathrm{mM}$ glyoxal, as assessed by real-time PCR (Figure 4A). Furthermore, this finding was supported by the presence of small dense particles on the surface of the cells, indicating secretory activity as shown by the immunostaining with antibody against EPO (Figure 4B). On the other hand, the expression of EPOR was heightened gradually under the influence of varying concentrations of glyoxal and further increased in the presence of exogenous EPO (Figure 4C). The increase in both EPO and EPOR expression suggested 

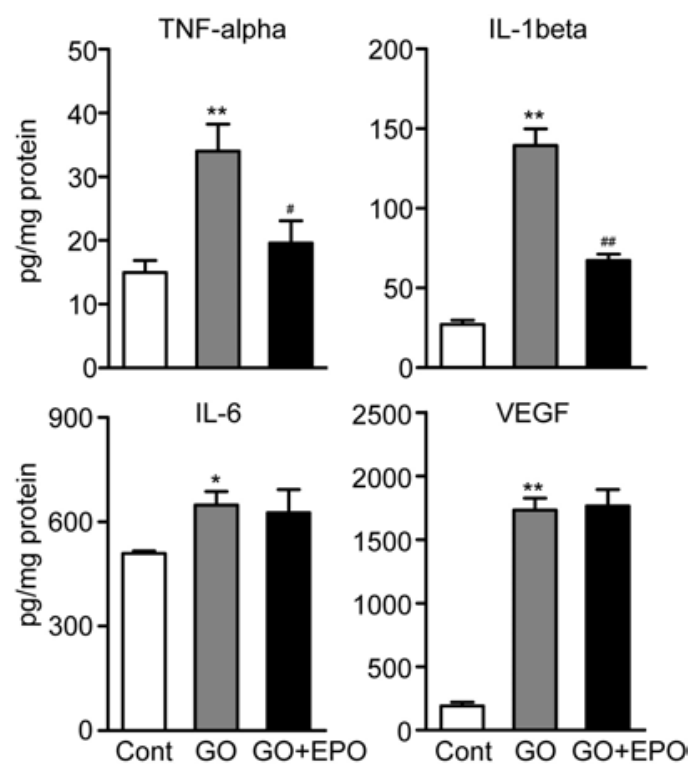

Figure 3. Effects of EPO on the secretion of the inflammatory cytokines in rMC-1 cells. The cytokines in supernatants of the cells exposed to $1 \mathrm{mM}$ glyoxal alone or glyoxal with $2 \mathrm{U} / \mathrm{ml}$ EPO were measured by ELISA and normalized to the protein content in the corresponding cells quantified by BCA assay. $\left({ }^{*} P<0.05, * * P<0.01\right.$ vs Cont; $\# P<0.05, \# \# P<0.01$ vs $\mathrm{GO} ; \mathrm{n}=4$.
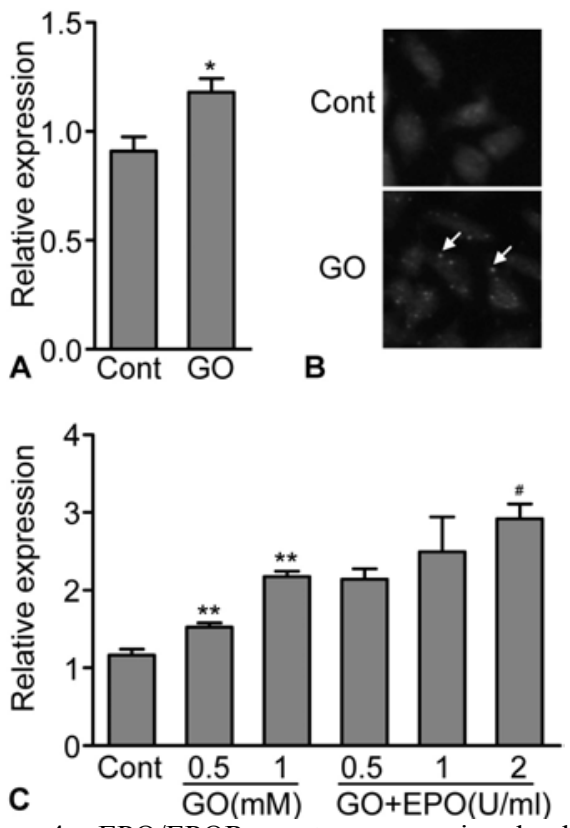

Figure 4. EPO/EPOR system was involved in the suppression effects of EPO on inflammatory genes in rMC1 cells. A: Elevated expression of EPO was detected by real-time PCR. $\left({ }^{*} P<0.05, n=4\right)$ B: An increase in secreted particles (arrow) on cell surface was shown by anti-EPO antibody immunostaining. Magnification: $\times 200$. C: Dosedependent elevation in EPOR mRNA expression by glyoxal injury and enhancement with the simultaneous addition of EPO were demonstrated by real-time PCR. $(* * P<0.01 v s$ Cont; $\# P<0.05$ vs 1 mM GO; $\mathrm{n}=4$ ) their significant correlation to the inflammatory response. When using sEPOR to block the effect of endogenous and exogenous EPO, no obvious changes were found in the expression levels of TNF-alpha (1.4 vs 1.5) and IL-1beta (1.4 vs 1.5 folds) after glyoxal treatment. However, an increase was observed (TNF-alpha, 1.4 vs 1.2; IL-1beta, 1.7 vs 1.1 folds) after glyoxal and EPO combined treatment (Figure 6).

\subsection{Modulation of AP-1 but not NF-kappaB activity by glyoxal and EPO}

As transcription factors AP-1 and NF-kappaB are essential regulators of several genes involved in the inflammatory response, we inspected whether they were modulated in our cell model. Results of luciferase assay revealed that the activity of AP-1 (175.7 vs 95.6) but not NF-kappaB (330.5 vs 306.3) was induced by glyoxal, and the highly activated AP-1 was down-regulated by EPO (117.3) (Figure 5A). Furthermore, it was confirmed that NF-kappaB was not involved in this process due to the lack of nuclear immunostaining for the NF-kappaB family member p65 after glyoxal exposure (Figure 5B).

NDGA inhibits lipoxygenase, which in turn blocks arachidonic acid metabolites and thus suppresses expression of the c-Fos component of AP-1. Pretreatment of rMC-1 cells with this AP-1 inhibitor abrogated TNFalpha and IL-1beta mRNA induction by glyoxal and mRNA inhibition by EPO (Figure 6). Therefore, these results confirmed that the inhibitory effects of EPO in this inflammatory response were carried out by the blockade of AP-1 activity induced by glyoxal.

\subsection{Attenuation of TNF-alpha and IL-1beta production by EPO in diabetic rats}

As all of the above results were obtained from the in vitro models, we performed an in vivo study using the STZinduced diabetic rats to further demonstrate the inhibitory effects of EPO on inflammatory reaction in the retinas. Two or twenty weeks after diabetes induction, rats received an intravitreal injection of EPO (8 $\mathrm{mU}$ per eye) or normal saline as control. $24 \mathrm{~h}$ following the operation, the rats were killed and the expression levels of TNF-alpha, IL-1beta, IL6 and VEGF in the retinas were determined by ELISA (Figure 7). In the two-week diabetic group, all of these cytokines were markedly increased except for VEGF (TNF-alpha, 4.7 vs 2.6; IL-1beta, 3.7 vs 0.8; IL-6, 43.3 vs $26.6 \mathrm{pg} / \mathrm{mg}$ protein, respectively). Furthermore, in the EPO treatment group, the production of TNF-alpha (3.0) and ILlbeta (1.3) was substantially decreased and IL-6 (31.6 $\mathrm{pg} / \mathrm{mg}$ protein) was also somewhat down-regulated. In addition, in the twenty-week diabetic group, similar changes were observed except for the moderate elevation of VEGF in diabetic retinas (78 vs $50 \mathrm{pg} / \mathrm{mg}$ protein). These in vivo results confirmed the inhibitory effects of EPO on TNF-alpha and IL-1beta production.

To investigate whether Muller cells were the main target cells of therapeutic EPO in rat retina, we conducted double labeling experiments using anti-IL-1beta and anti-CRALBP antibodies on cryostat sections. In the twenty-week diabetic rat retinas, anti-IL-1beta staining 


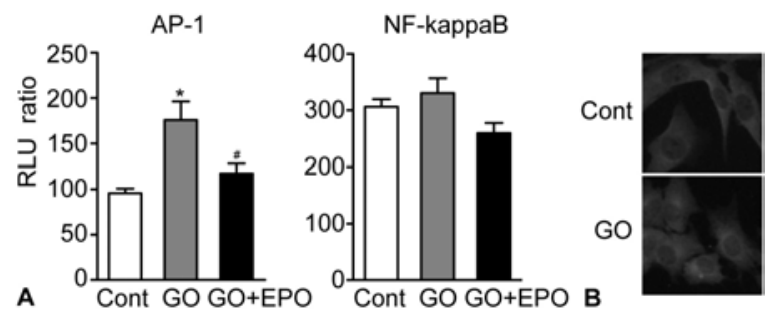

Figure 5. Altered activity of transcription factor AP-1, but not NF-kappaB was associated with the inhibitory effects of EPO on inflammatory genes. A: Induction of AP-1 or NF-kappaB was determined individually using Dual Luciferase Assay System in rMC-1 cells co-transfected with reporter gene plasmid (pNF-kappaB-luc or pAP1-luc) and internal control plasmid (pRL-CMV) before treatment with either $1 \mathrm{mM}$ glyoxal or glyoxal with $2 \mathrm{U} / \mathrm{ml}$ EPO. $\left({ }^{*} P<0.05\right.$ vs Cont; $\# P<0.05$ vs $1 \mathrm{mM} \mathrm{GO}$; $\left.\mathrm{n}=4\right) \mathrm{B}$ : Nuclear translocation of the NF-kappaB family member p65 was not detected by anti-p65 antibody immunostaining after 1 $\mathrm{mM}$ glyoxal exposure for $12 \mathrm{~h}$. Magnification: $\times 200$.
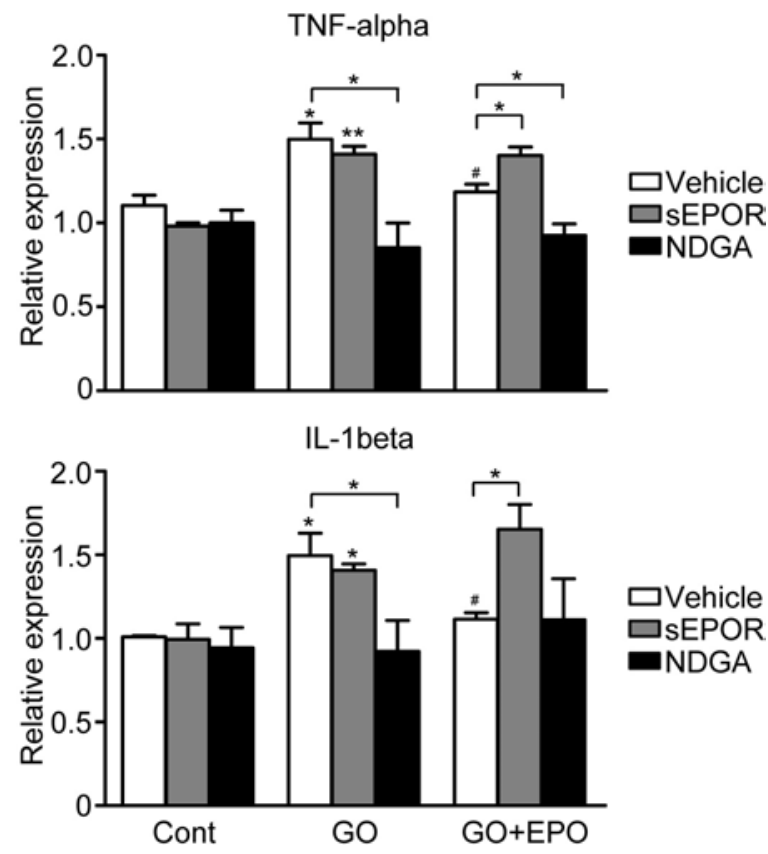

Figure 6. The modulation of TNF-alpha and IL-1beta expression by glyoxal and EPO in rMC-1 cells were altered by SEPOR or AP-1 inhibitor NDGA pretreatment for $1 \mathrm{~h}$ before the stimulation as detected by real-time PCR. $\left({ }^{*} P<0.05, \quad * * P<0.01\right.$ vs Cont or the indicated group; $\# P<0.05$ vs 1 mM GO; $\mathrm{n}=4)$

became more intense compared with control retina, especially in the ganglion cell layer, inner plexiform layer and inner nuclear layer (Figure 8A). With EPO injection, the staining in all the three layers decreased. Similar to these changes, a much deeper staining of anti-CRALBP was obtained in diabetic retina, notably in the end feet of the cells in ganglion cell layer and throughout the plexiform layer, whereas less dense staining was found in EPO treatment retina. In addition, the co-localization of
anti-IL-1beta with anti-CRALBP was mainly observed within the end feet of Muller cells in ganglion cell layer (Figure 8B). These results indicated that Muller cells were the main cellular origin of IL-1beta and the most prominent target cells for EPO therapy in diabetic retina, though astrocytes, microglia and endothelial cells could not be completely ruled out.

\section{DISCUSSION}

Apart from its hematopoietic effect, EPO is known as a pleiotropic cytokine with anti-inflammatory and antiapoptotic properties (28). The present study focused on the anti-inflammatory function of EPO and revealed that it could attenuate the increase of TNF-alpha and IL-1beta but not IL-6 and VEGF in cell culture and animal models under diabetes-like conditions. Furthermore, our results demonstrated that the modulation effects of EPO occur through inhibition upon the transcription factor AP-1.

Increasing evidence suggests that the pathophysiology of diabetes is considerably analogous to chronic inflammatory states (29). It is well known that high serum glucose can lead to nonenzymatic binding of glucose to protein side chains, resulting in the formation of AGEs. Therefore, the intermediates of this reaction, methylglyoxal and glyoxal, may have the function of influencing the production of inflammatory factors (30-32). It is reported that prolonged exposure of rats to methylglyoxal induced pro-inflammatory responses, including increased TNFalpha and IL-1beta expression in granulation tissue during cutaneous wound healing (30). Methylglyoxal also caused a very significant increase in both transcript and protein expression of IL-1beta in cultured hippocampal neuronal cells (31). Recently, a study proved that both methylglyoxal and glyoxal could induce a concentration dependent enhancement of IL-6 secretion in intestinal cell line (32). It has been shown that VEGF was increased in cultured rat mesothelial and human endothelial cells exposed to methylglyoxal, but glyoxal could not modify VEGF expression in mesothelial cells (33). Here we reported that all four of these cytokines were elevated substantially in rat Muller cells stimulated with glyoxal at non-cytotoxic concentrations, specifically $1 \mathrm{mM}$ (Figure 2). As shown in real-time PCR results, the induction of TNF-alpha and ILlbeta with similar pattern suggested the same signaling pathway was involved, which may be different from the pathways associated with IL-6 or VEGF production. In our results, the induction of AP-1 but not NF-kappaB was demonstrated by luciferase assay (Figure 5A). And nuclear translocation of the NF-kappaB family member p65 was not detected after glyoxal exposure (Figure 5B), which is similar to the observation in human vascular endothelial cells after glyoxal treatment (5). Using the AP-1 inhibitor NDGA, the induction of TNF-alpha and IL-1beta could not be found any more (Figure 6). These findings document the involvement of AP-1 transcription factor in this process.

Even though the anti-inflammatory effects of EPO have been shown by many studies, the underlying molecular mechanisms remain to be elucidated. The present results demonstrate the direct inhibitory effect of EPO on 

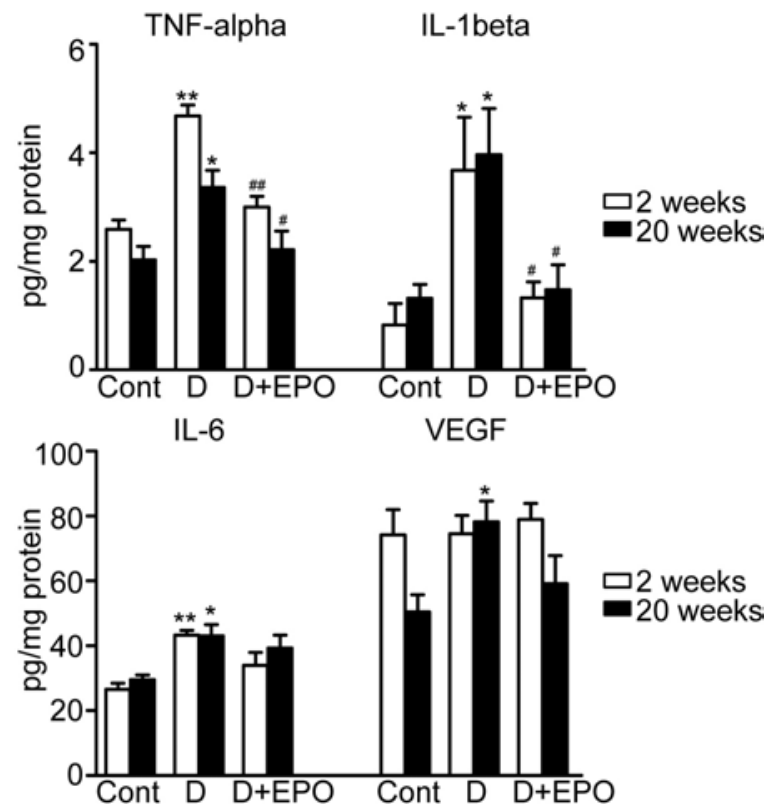

Figure 7. The inhibitory effects of EPO on inflammatory cytokines in STZ-induced diabetic rat retinas. Expression levels of the inflammatory genes were measured by ELISA in the rat retinas from age-matched control, diabetic (D) and diabetic eyes with EPO intravitreal injection (D+EPO) using two- or twenty-week models. $\left({ }^{*} P<0.05,{ }^{*} P<0.01 \mathrm{vs}\right.$ Cont; $\# P<0.05, \# \# P<0.01$ vs $\mathrm{D} ; \mathrm{n}=6$ )

inflammatory cytokines TNF-alpha and IL-1beta (Figure 3). Similarly, it has been reported by others that EPO treatment decreased TNF-alpha secretion in undifferentiated U937 human histiocytic lymphoma cells (23). Besides, our results showed no reduction by EPO on IL-6 and VEGF secretion, whereas EPO exerted the inhibitory effect on IL-6 production in U937 cells at the $10 \mathrm{IU} / \mathrm{ml}$ dosage (23). With regard to the signaling pathway possibly involved in this effect, both AP-1 and NF-kappaB were speculated as the candidates. The down-regulation by EPO on the activity of AP-1 was detected more apparently in the present study (Figure 5A). Nevertheless, previous studies revealed that EPO pretreatment could suppress the activation of NFkappaB in cardiomyocytes exposed to hypoxia/reoxygenation injury through a negative feedback of NF-kappaB signaling pathway (24). These differences in anti-inflammatory effects of EPO may be related to the severity of injury, the doses administered, or also may depend on different cell types. We propose that EPO attenuates glyoxal-induced AP-1 activation resulting in decrease of TNF-alpha and IL-1beta production. IL- 6 and VEGF were also increased by glyoxal, but EPO had no effect on these increases in expression.

Endogenous EPO/EPOR system appears to be involved in the regulation of inflammatory cytokines. Muller cells were verified as the main source of EPO in the normal retina (27) and EPO autocrine loop was found in various cells (34-36). It is critical to determine whether EPO and EPOR are altered in Muller cells after injury. Previous in vitro studies showed EPO expression in astrocytes was down-regulated when exposed to inflammatory cytokines such as IL-1beta, IL-6 and TNF-alpha (37). In the present study, after glyoxal treatment, both EPO and EPOR were elevated, and EPOR was increased more significantly (Figure 4). The possible reason for that the increased EPO in a much smaller scale is due to the inhibitory effects by glyoxalinduced cytokines. No increase was found in TNF-alpha or IL-1beta mRNA level after blocking endogenous EPO by sEPOR after glyoxal treatment (Figure 6). Therefore, the enhanced expression of EPOR along with increased EPO production indicates the demand for protection from cells after inflammatory injury (19). The active response of EPO/EPOR expression in Muller cells to exogenous EPO after inflammation provided evidence of a glial paracrine protective function on neighbouring neurons.

Our previous study demonstrated an ameliorating effect of EPO on BRB breakdown in STZinduced diabetic rats (19). In the present experiments using two-week diabetic rats, marked elevations in TNFalpha, IL-1beta and IL-6 levels were detected, and no significant increase in VEGF production was observed in the retinas (Figure 7), which might give us insights into the relative contributions of TNF-alpha, IL-1beta and IL- 6 to the early increased BRB permeability. However, in twenty-week diabetic rats, VEGF was increased moderately and $8 \mathrm{mU} /$ eye EPO treatment could down-regulate it slightly, which is consistent with our previous report (38) (Figure 7). Furthermore, after EPO intravitreal injection, TNF-alpha and IL-1beta expression could be down-regulated, while IL-6 production was basically not changed at the same diabetic course. Similarly, no apparent effect on IL-6 after EPO treatment was also reported in animal models of traumatic brain injury (39). Most interestingly, the inhibitory effect of EPO was in parallel with a marked decrease in Muller glial activation, as assessed by antiIL-1beta and anti-CRALBP immunostaining (Figure 8). The most likely source of IL-1beta was Muller cells, which was denoted by the co-localization, mainly at the cell processes encasing ganglion cells. In fact, other retinal cell types (e.g. microglia) also release inflammatory cytokines in response to diabetic stress (40). Upon their release, the cytokines could propagate the inflammatory response within the retina, leading to increased leukocyte infiltration and neuronal cell death (41). The activated Muller cells and their secretion of inflammatory factors may be crucial contributors to the increased death of neurons and the aggravated breakdown of BRB in these diseases. Therefore, the suppression of TNF-alpha and IL-1beta expression by EPO in Muller cells could be a therapeutic approach for DR.

In summary, exogenously administered EPO confers a strong anti-inflammatory effect within the setting of experimental diabetic retinopathy mainly through the suppression of TNF-alpha and IL-1beta 


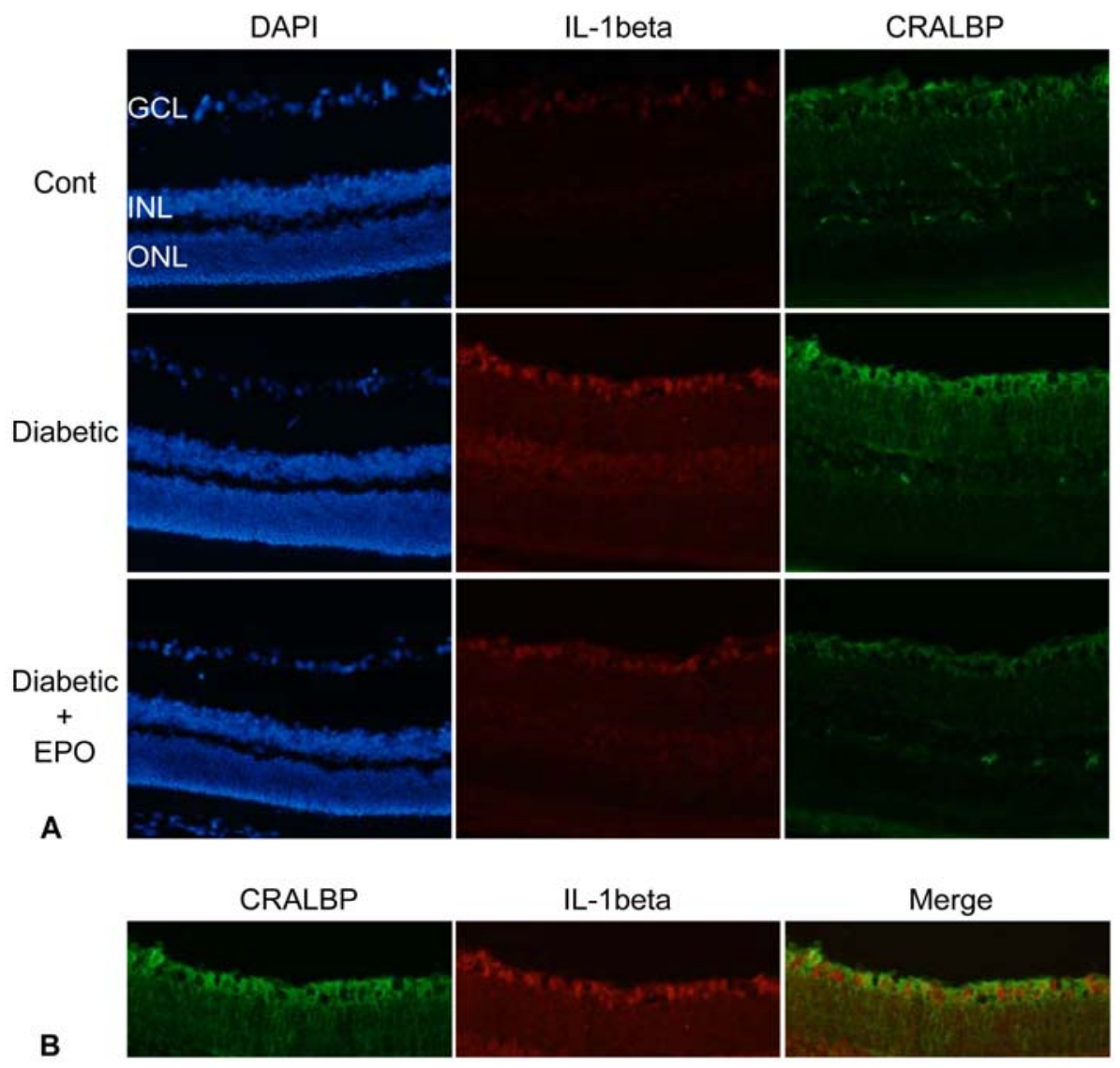

Figure 8. Double immunostaining with anti-IL-1beta and anti-CRALBP antibodies on cryostat sections of the twenty-week diabetic rat retinas. A: Representative photomicrographs of the sections from the control, diabetic or diabetic retinas with EPO treatment labeled with DAPI (blue), anti-IL-1beta (red) and anti-CRALBP (green); B: Merged images from the diabetic group demonstrated that Muller cell processes encasing ganglion cells exhibited the highest IL-1beta and CRALBP co-localization (yellow). GCL: ganglion cell layer; INL: inner nuclear layer; ONL: outer nuclear layer. Magnification: $\times 200$.

expression. However, the production of IL-6 and VEGF is not overtly affected by exposure to EPO. Our data support a model wherein EPO attenuates the production of inflammatory cytokines by Muller cells via the regulation of the transcription factor AP-1 rather than NF-kappaB, which in turn, results in a protection of the BRB integrity and retinal cell survival.

\section{ACKNOWLEDGMENT}

Drs Weiye $\mathrm{Li}$ and Guo-Tong $\mathrm{Xu}$ are cocorresponding authors who contributed equally to this study. This project is supported by Sciences and Technology Commission of Shanghai Municipality: 08ZR1422100 and 08410701200.

\section{REFERENCES}

1. D. S. Fong, L. P. Aiello, F. L. Ferris, 3rd and R. Klein: Diabetic retinopathy. Diabetes Care 27, 2540-53 (2004)

2. J. L. Wautier and P. J. Guillausseau: Advanced glycation end products, their receptors and diabetic angiopathy. Diabetes Metab 27, 535-42 (2001)
3. J. Shen, Y. Wu, J. Y. Xu, J. Zhang, S. H. Sinclair, M. Yanoff, G. Xu, W. Li and G. T. Xu: ERK- and Aktdependent neuroprotection by erythropoietin (EPO) against glyoxal-AGEs via modulation of Bcl-xL, Bax, and BAD. Invest Ophthalmol Vis Sci 51, 35-46 (2009)

4. E. M. Kniep, C. Roehlecke, N. Ozkucur, A. Steinberg, F. Reber, L. Knels and R. H. Funk: Inhibition of apoptosis and reduction of intracellular $\mathrm{pH}$ decrease in retinal neural cell cultures by a blocker of carbonic anhydrase. Invest Ophthalmol Vis Sci 47, 1185-92 (2006)

5. H. Yamawaki and Y. Hara: Glyoxal causes inflammatory injury in human vascular endothelial cells. Biochem Biophys Res Commun 369, 1155-9 (2008)

6. A. M. Joussen, V. Poulaki, N. Mitsiades, B. Kirchhof, K. Koizumi, S. Dohmen and A. P. Adamis: Nonsteroidal antiinflammatory drugs prevent early diabetic retinopathy via TNF-alpha suppression. Faseb J 16, 438-40 (2002)

7. A. Carmo, J. G. Cunha-Vaz, A. P. Carvalho and M. C. Lopes: Effect of cyclosporin-A on the blood--retinal barrier 
permeability in streptozotocin-induced diabetes. Mediators Inflamm 9, 243-8 (2000)

8. H. Funatsu, H. Yamashita, E. Shimizu, R. Kojima and S. Hori: Relationship between vascular endothelial growth factor and interleukin-6 in diabetic retinopathy. Retina 21, 469-77 (2001)

9. T. Qaum, Q. Xu, A. M. Joussen, M. W. Clemens, W. Qin, K. Miyamoto, H. Hassessian, S. J. Wiegand, J. Rudge, G. D. Yancopoulos and A. P. Adamis: VEGF-initiated blood-retinal barrier breakdown in early diabetes. Invest Ophthalmol Vis Sci 42, 2408-13 (2001)

10. R. J. Walker and J. J. Steinle: Role of beta-adrenergic receptors in inflammatory marker expression in Muller cells. Invest Ophthalmol Vis Sci 48, 5276-81 (2007)

11. M. D. Shelton, T. S. Kern and J. J. Mieyal: Glutaredoxin regulates nuclear factor kappa-B and intercellular adhesion molecule in Muller cells: model of diabetic retinopathy. J Biol Chem 282, 12467-74 (2007)

12. Y. Xiong, D. Lu, C. Qu, A. Goussev, T. Schallert, A. Mahmood and M. Chopp: Effects of erythropoietin on reducing brain damage and improving functional outcome after traumatic brain injury in mice. $J$ Neurosurg 109, 510 $21(2008)$

13. P. Villa, P. Bigini, T. Mennini, D. Agnello, T. Laragione, A. Cagnotto, B. Viviani, M. Marinovich, A. Cerami, T. R. Coleman, M. Brines and P. Ghezzi: Erythropoietin selectively attenuates cytokine production and inflammation in cerebral ischemia by targeting neuronal apoptosis. J Exp Med 198, 971-5 (2003)

14. M. L. Brines, P. Ghezzi, S. Keenan, D. Agnello, N. C. de Lanerolle, C. Cerami, L. M. Itri and A. Cerami: Erythropoietin crosses the blood-brain barrier to protect against experimental brain injury. Proc Natl Acad Sci US A 97, 10526-31 (2000)

15. M. Celik, N. Gokmen, S. Erbayraktar, M. Akhisaroglu, S. Konakc, C. Ulukus, S. Genc, K. Genc, E. Sagiroglu, A. Cerami and M. Brines: Erythropoietin prevents motor neuron apoptosis and neurologic disability in experimental spinal cord ischemic injury. Proc Natl Acad Sci U S A 99, 2258-63 (2002)

16. D. Agnello, P. Bigini, P. Villa, T. Mennini, A. Cerami, M. L. Brines and P. Ghezzi: Erythropoietin exerts an antiinflammatory effect on the CNS in a model of experimental autoimmune encephalomyelitis. Brain Res 952, 128-34 (2002)

17. A. Gorio, N. Gokmen, S. Erbayraktar, O. Yilmaz, L. Madaschi, C. Cichetti, A. M. Di Giulio, E. Vardar, A. Cerami and M. Brines: Recombinant human erythropoietin counteracts secondary injury and markedly enhances neurological recovery from experimental spinal cord trauma. Proc Natl Acad Sci U S A 99, 9450-5 (2002)
18. C. Savino, R. Pedotti, F. Baggi, F. Ubiali, B. Gallo, S. Nava, P. Bigini, S. Barbera, E. Fumagalli, T. Mennini, A. Vezzani, M. Rizzi, T. Coleman, A. Cerami, M. Brines, P. Ghezzi and R. Bianchi: Delayed administration of erythropoietin and its non-erythropoietic derivatives ameliorates chronic murine autoimmune encephalomyelitis. J Neuroimmunol 172, 27-37 (2006)

19. J. Zhang, Y. Wu, Y. Jin, F. Ji, S. H. Sinclair, Y. Luo, G. $\mathrm{Xu}$, L. Lu, W. Dai, M. Yanoff, W. Li and G. T. Xu: Intravitreal injection of erythropoietin protects both retinal vascular and neuronal cells in early diabetes. Invest Ophthalmol Vis Sci 49, 732-42 (2008)

20. W. Li, S. H. Sinclair and G. T. Xu: Effects of Intravitreal Erythropoietin Therapy for Patients with Chronic and Progressive Diabetic Macular Edema. Ophthalmic Surgery, Lasers and Imaging 41, 18-25 (2010)

21. T. S. Kern: Contributions of inflammatory processes to the development of the early stages of diabetic retinopathy. Exp Diabetes Res 2007, 95103 (2007)

22. X. Liu, W. Xie, P. Liu, M. Duan, Z. Jia, W. Li and J. $\mathrm{Xu}$ : Mechanism of the cardioprotection of rhEPO pretreatment on suppressing the inflammatory response in ischemia-reperfusion. Life Sci 78, 2255-64 (2006)

23. N. Yazihan, O. Karakurt and H. Ataoglu: Erythropoietin reduces lipopolysaccharide-induced cell Damage and midkine secretion in U937 human histiocytic lymphoma cells. Adv Ther 25, 502-14 (2008)

24. C. Qin, Y. B. Xiao, Q. J. Zhong, L. Chen and X. F. Wang: Anti-inflammatory effect of erythropoietin pretreatment on cardiomyocytes with hypoxia/reoxygenation injury and the possible mechanism. Chin J Traumatol 11, 352-8 (2008)

25. W. M. Campana, X. Li, V. I. Shubayev, M. Angert, K. Cai and R. R. Myers: Erythropoietin reduces Schwann cell TNF-alpha, Wallerian degeneration and pain-related behaviors after peripheral nerve injury. Eur J Neurosci 23, 617-26 (2006)

26. Z. Y. Wang, L. J. Shen, L. Tu, D. N. Hu, G. Y. Liu, Z. L. Zhou, Y. Lin, L. H. Chen and J. Qu: Erythropoietin protects retinal pigment epithelial cells from oxidative damage. Free Radic Biol Med 46, 1032-41 (2009)

27. Q. L. Fu, W. Wu, H. Wang, X. Li, V. W. Lee and K. F. So: Up-regulated endogenous erythropoietin/erythropoietin receptor system and exogenous erythropoietin rescue retinal ganglion cells after chronic ocular hypertension. Cell Mol Neurobiol 28, 317-29 (2008)

28. M. Brines and A. Cerami: Emerging biological roles for erythropoietin in the nervous system. Nat Rev Neurosci 6, 484-94 (2005)

29. S. F. Yan, R. Ramasamy, Y. Naka and A. M. Schmidt: Glycation, inflammation, and RAGE: a scaffold for the 
macrovascular complications of diabetes and beyond. Circ Res 93, 1159-69 (2003)

30. J. Berlanga, D. Cibrian, I. Guillen, F. Freyre, J. S. Alba, P. Lopez-Saura, N. Merino, A. Aldama, A. M. Quintela, M. E. Triana, J. F. Montequin, H. Ajamieh, D. Urquiza, N. Ahmed and P. J. Thornalley: Methylglyoxal administration induces diabetes-like microvascular changes and perturbs the healing process of cutaneous wounds. Clin Sci (Lond) $109,83-95(2005)$

31. S. Di Loreto, V. Caracciolo, S. Colafarina, P. Sebastiani, A. Gasbarri and F. Amicarelli: Methylglyoxal induces oxidative stress-dependent cell injury and upregulation of interleukin-1beta and nerve growth factor in cultured hippocampal neuronal cells. Brain Res 1006, 15767 (2004)

32. S. Kuntz, S. Rudloff, J. Ehl, R. G. Bretzel and C. Kunz: Food derived carbonyl compounds affect basal and stimulated secretion of interleukin- 6 and -8 in Caco-2 cells. Eur J Nutr 48, 499-503 (2009)

33. R. Inagi, T. Miyata, T. Yamamoto, D. Suzuki, K. Urakami, A. Saito, C. van Ypersele de Strihou and K. Kurokawa: Glucose degradation product methylglyoxal enhances the production of vascular endothelial growth factor in peritoneal cells: role in the functional and morphological alterations of peritoneal membranes in peritoneal dialysis. FEBS Lett 463, 260-4 (1999)

34. J. Y. Jeong, G. Hoxhaj, A. L. Socha, A. J. Sytkowski and L. Feldman: An erythropoietin autocrine/paracrine axis modulates the growth and survival of human prostate cancer cells. Mol Cancer Res 7, 1150-7 (2009)

35. S. M. Kumar, G. Acs, D. Fang, M. Herlyn, D. E. Elder and X. Xu: Functional erythropoietin autocrine loop in melanoma. Am J Pathol 166, 823-30 (2005)

36. G. Acs, M. Chen, X. Xu, P. Acs, A. Verma and C. J. Koch: Autocrine erythropoietin signaling inhibits hypoxiainduced apoptosis in human breast carcinoma cells. Cancer Lett 214, 243-51 (2004)

37. A. Nagai, E. Nakagawa, H. B. Choi, K. Hatori, S. Kobayashi and S. U. Kim: Erythropoietin and erythropoietin receptors in human CNS neurons, astrocytes, microglia, and oligodendrocytes grown in culture. $J$ Neuropathol Exp Neurol 60, 386-92 (2001)

38. J. Zhang, L. M. Hu, G. Xu, Y. Wu, J. Shen, Y. Luo, Y. Zhong, S. H. Sinclair, M. Yanoff, W. Li and G. T. Xu: Anti-VEGF effects of intravitreal erythropoietin in early diabetic retinopathy. Front Biosci (2010, in press)

39. G. Chen, J. X. Shi, C. H. Hang, W. Xie, J. Liu and X. Liu: Inhibitory effect on cerebral inflammatory agents that accompany traumatic brain injury in a rat model: a potential neuroprotective mechanism of recombinant human erythropoietin (rhEPO). Neurosci Lett 425, 177-82 (2007)
40. A. L. Wang, Z. Li, M. Yuan, A. C. Yu, X. Zhu and M. O. Tso: Sinomenine inhibits activation of rat retinal microglia induced by advanced glycation end products. Int Immunopharmacol 7, 1552-8 (2007)

41. L. P. Yang, H. L. Sun, L. M. Wu, X. J. Guo, H. L. Dou, M. O. Tso, L. Zhao and S. M. Li: Baicalein reduces inflammatory process in a rodent model of diabetic retinopathy. Invest Ophthalmol Vis Sci 50, 2319-27 (2009)

Abbreviations: DR: diabetic retinopathy; EPO: erythropoietin; EPOR: erythropoietin receptor; TNF-alpha: tumor necrosis factor-alpha; IL-1beta: interleukin-1beta; IL-6: interleukin-6; VEGF: vascular endothelial growth factor; NF-kappaB: nuclear factor kappaB; AP-1: activator protein 1; CRALBP: cellular retinaldehyde-binding protein; GO: Glyoxal; AGEs: glycation end products; BRB: bloodretinal barrier; STZ: streptozotocin; sEPOR: soluble EPO receptor; NDGA: nordihydroguaiaretic acid

Key Words: Diabetic Retinopathy, Erythropoietin, Inflammatory Cytokines, TNF-alpha, IL-1beta, Muller Cells, Activator Protein 1

Send correspondence to: Guo-Tong $\mathrm{Xu}$, Tongji Eye Institute and Department of Regenerative Medicine, Tongji University School of Medicine, 1239 Siping Road, Medical School Building, Room 521, Shanghai, 200092, China, Tel: 86-21-6598-6358, Fax: 86-21-6598-6358, E-mail: gtxu@tongji.edu.cn 